\title{
Distributed Winner-Take-All Teleoperation of A Multi-Robot System
}

\author{
Yuan Yang, Daniela Constantinescu and Yang Shi
}

\begin{abstract}
In a distributed multi-master-multi-slave teleoperation system, the human users may compete against each other for the control of the team of slave robots. To win the competition, one operator would send the largest command to the slave group. For the sake of team cohesion, the slave group should follow the command of the winning operator and ignore the commands of the other users. To enable (i) the slave team to identify the winning operator, and (ii) each slave to determine whether to admit or discard the command it receives from its operator, this paper proposes a dynamic decision-making protocol that distinguishes the decision variable of the slave commanded by the winner from the decision variables of all other slave robots. The protocol only requires the slaves to exchange and evaluate their decision variables locally. Lyapunov stability analysis proves the theoretical convergence of the proposed decision-making algorithm. An experimental distributed winner-take-all teleoperation in a 3-masters-11-slaves teleoperation testbed validates its practical efficacy.
\end{abstract}

\section{INTRODUCTION}

Multi-robot systems teleoperated by human operators can avail of the human intelligence when making critical decisions and when having to respond fast to unexpected situations [1]. Yet, the interaction between cooperative robot teams and human operators may become unstable [2]. Being sufficient for stabilizing robot teams in interaction with passive users, the passivity of the robot team has become a prevalent design objective in teleoperation control [3], [4].

Numerous passivity-based control techniques have tackled slave collisions with other slaves and the remote environment, limited robot communication ranges, and master-slave kinematic dissimilarities [5]. An energy tank-based strategy has created and broken slave interconnections to enable a teleoperated robot group to passively avoid obstacles [6]. A potential function of the estimated algebraic connectivity has provided a decentralized controller for global connectivity preservation of a teleoperated robot team [7]. A hierarchical control using energy tanks with energy flows assigned by port-Hamiltonian analysis has overcome kinematic dissimilarity [8].

Recent research has started to control multi-robot systems by optimizing their interaction with external terminators subject to system passivity. In [9], a quadratic optimization constrained by proper scaling of inter-robot connections has regulated passive interaction forces between a robot group and external entities. In [10], an energy tank-based optimization has adapted the admittance of the robot team subject to a condition, on the lower bound of energy storage, guaranteeing passivity. In [11], a generalized two-layer approach has let all masters/slaves share a single energy tank to minimize the conservativeness of a maintaining the multi-master-multi-slave teleoperator passive. In [12], an energy transaction protocol has distributed energy budgets passively in the multi-robot system.

The aforementioned works share a lower-level control objective: to render a teleoperated multi-robot system passive. Yet, multi-user teleoperation introduces an additional higherlevel decision-making problem rarely covered [13]: a multislave team must determine how to respond to simultaneous commands from multiple users [14]. Relevant work has mainly tracked the average of the multiple exogenous inputs (user commands in multi-user teleoperation). In [15], a filter-based algorithm with state-dependent gains has enabled dynamic average tracking control of nonlinear second-order multi-agent systems. In [16], a robust approach has guaranteed arbitrarily small steady-state error of dynamic average consensus for directed networks. In [17], an integral-based dynamic consensus technique has robustly filtered the average of only nonzero sensor measurements.

This paper introduces a distributed decision-making mechanism that enables a teleoperated robot group to identify, and carry out the commands of only, the winner among its multiple operators. Inspired by Oja's rule for principal component analysis [18], the proposed strategy endows each slave with a decision variable whose evolution depends on (i) the input from its local operator and (ii) the decision variables of its neighbouring slaves. Compared to [19], the distributed decision layer can handle the time-varying operator commands arising in teleoperation by exploiting robust dynamic average estimations. Lyapunov energy analysis proves that the decision variables of all slaves in communication with the winner converge to a positive limit set and all other decision variables asymptotically approach zero when the winner is unique. Thus, it shows that the proposed decision-making method enables a teleoperated multi-robot team to distinguish the winner among its multiple human users by evaluating only local decision variables. Then, letting only the slaves with decision variables above a properly selected threshold to apply their received operator commands establishes winner-take-all teleoperation: the slave team is teleoperated exclusively by the winner. An experimental winner-take-all teleoperation in a 3-masters-11slaves testbed validates the practical efficacy of the distributed decision-making algorithm.

\section{PROBLEM Formulation}

Let a multi-user teleoperation system include $N_{m}>1$ masters and $N_{s} \geq N_{m}$ slaves, all gravity compensated. 
Without loss of generality, let the slave team be connected and each slave receive information from at most one master.

The task-space dynamics of the master robots are:

$$
\mathbf{M}_{m i}\left(\mathbf{y}_{m i}\right) \cdot \ddot{\mathbf{y}}_{m i}+\mathbf{C}_{m i}\left(\mathbf{y}_{m i}, \dot{\mathbf{y}}_{m i}\right) \cdot \dot{\mathbf{y}}_{m i}=\mathbf{f}_{h i}-\mathbf{u}_{m i},
$$

where $i=1, \cdots, N_{m}$ indexes all human users and their master devices. For each master $i: \mathbf{y}_{m i}, \dot{\mathbf{y}}_{m i}$ and $\ddot{\mathbf{y}}_{m i}$ are its position, velocity and acceleration; $\mathbf{M}_{m i}\left(\mathbf{y}_{m i}\right), \mathbf{C}_{m i}\left(\mathbf{y}_{m i}, \dot{\mathbf{y}}_{m i}\right)$ are its matrices of inertia and of Coriolis and centrifugal effects; $-\mathbf{u}_{m i}$ and $\mathbf{f}_{h i}$ are the forces applied by its own controller and by its user, respectively. The control forces:

$$
\mathbf{u}_{m i}=K_{m p} \mathbf{y}_{m i}+K_{m d} \dot{\mathbf{y}}_{m i}
$$

with $K_{m p}$ and $K_{m d}$ well-tuned positive gains, (i) provide force feedback to the operators and (ii) transmit the operator commands to the $N_{l s}$ active slaves, where $N_{m} \leq N_{l s} \leq N_{s}$.

To synchronize the slaves: (i) connect each slave to an own second-order virtual proxy; and (ii) couple the proxies of adjacent slaves. Then, the slave group dynamics become:

$$
\begin{array}{r}
\hat{m}_{s i} \ddot{\hat{\mathbf{y}}}_{s i}=\sum_{j \sim i} \hat{K}_{s p}\left(\hat{\mathbf{y}}_{s j}-\hat{\mathbf{y}}_{s i}\right)-\hat{K}_{s d} \dot{\hat{\mathbf{y}}}_{s i}+\mathbf{f}_{s i}, \\
\mathbf{M}_{s i}\left(\mathbf{y}_{s i}\right) \cdot \ddot{\mathbf{y}}_{s i}+\mathbf{C}_{s i}\left(\mathbf{y}_{s i}, \dot{\mathbf{y}}_{s i}\right) \cdot \dot{\mathbf{x}}_{s i}=K_{s p} \tilde{\mathbf{y}}_{s i}-K_{s d} \dot{\mathbf{y}}_{s i},
\end{array}
$$

where the index $i=1, \cdots, N_{l s}$ indicates the active slaves, i.e., slaves that receive user commands from masters, $i=$ $N_{l s}+1, \cdots, N_{s}$ indicates asleep slaves, i.e., slaves that communicate only to other slaves, and $j \sim i$ indicates the set of neighbours of the slave $i$, i.e., slaves $j$ that exchange information with slave $i$. For every slave $i: \hat{m}_{s i}, \hat{\mathbf{y}}_{s i}, \dot{\hat{\mathbf{y}}}_{s i}$ and $\hat{\mathbf{y}}_{s i}$ are the mass, position, velocity and acceleration of its virtual proxy; $\tilde{\mathbf{y}}_{s i}=\hat{\mathbf{y}}_{s i}-\mathbf{y}_{s i}$ is the displacement of its proxy from it; and $\mathbf{M}_{s i}\left(\mathbf{y}_{s i}\right), \mathbf{C}_{s i}\left(\mathbf{y}_{s i}, \dot{\mathbf{y}}_{s i}\right), \mathbf{y}_{s i}, \dot{\mathbf{y}}_{s i}$ and $\ddot{\mathbf{y}}_{s i}$ are the same quantities as in (1) but for slave robots; and $\mathbf{f}_{s i}$ is determined by the proposed decision-making strategy. The positive gains $\hat{K}_{s p}, \hat{K}_{s d}, K_{s p}$ and $K_{s d}$ are tuned for stable and tight coordination of the slave network, similar to [20].

The proposed decision-making strategy associates a decision variable $x_{i}$ with each slave and determines $\mathbf{f}_{s i}$ in (3) as follows: $\mathbf{f}_{s i}=\mathbf{0}$ for each asleep slave $i=N_{l s}+1, \cdots, N_{s}$, because asleep slaves communicate with no master; $\mathbf{f}_{s i}=\mathbf{u}_{m j}$ for the active slave $i$ commanded by the winner operator $j$, i.e., the active slave with decision variable $x_{i}$ above a threshold; and $\mathbf{f}_{s i}=\mathbf{0}$ for all active slaves $i$ who communicate with the remaining users, i.e., active slaves with decision variables $x_{i}$ below the threshold. Identifying the winner among all users then lies in evolving the decision variables $x_{i}$ appropriately. The decision-making mechanism evolving $x_{i}$ is the primary concern of this paper.

In the absence of a decision-making strategy, all users can teleoperate the slave team from their different locations. Their commands $\mathbf{u}_{m i}$ can stand for the degree of their demands to steer the slave group: user $i$ may increase their command $\mathbf{u}_{m i}$ when they intend to gain more control of the slave network. In Figure 1, for example, users $i=1,2,3$ all send commands to the slave team and may compete to dominate the teleoperation of the multi-slave group. Suppose all operator commands are applied to the slave network, i.e., all switches are closed. If user 1 sought to control the network regardless of opposition from users 2 and 3, user 1 would keep increasing their command $\mathbf{u}_{m 1}$ to conquer $\mathbf{u}_{m 2}$ and $\mathbf{u}_{m 3}$, while users 2 and 3 would slow the movement of the slave team and threaten its coordination.

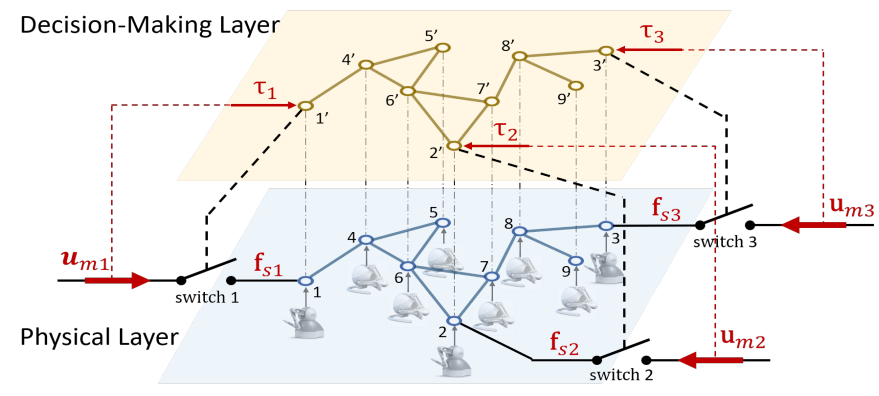

Figure 1. A slave robot team, with 3 active slaves (Geomagic Touch robots) and 6 asleep slaves (Novint Falcon robots), teleoperated by 3 users.

To guarantee the agility and cohesion of the slave group, this paper proposes winner-take-all teleoperation, which permits only the winner user to teleoperate the slave network. Winnertake-all teleoperation requires a decision-making mechanism that enables all slaves to recognize the winner and ignore the commands of all other operators. Hence, this paper develops a distributed decision-making protocol that permits each slave to detect whether it receives commands from the winner by evaluating its own decision variable based only on information from its neighbouring slaves.

The proposed protocol endows each slave $i$ with a dynamic decision variable $x_{i}$ and builds a decision-making layer with inputs $\tau_{i}$ that encode the received operator commands $\mathbf{u}_{m j}$. This paper uses the example encoding:

$$
\tau_{i}=\min \left[\exp \left(\left\|\mathbf{u}_{m j}\right\| / 5\right), \bar{\tau}\right],
$$

where $i=1, \cdots, N_{s}, j=1, \cdots, N_{m}, \bar{\tau}>1$ and $\mathbf{u}_{m j}$ is given in (2) for $i=1, \cdots, N_{l s}$ and $\mathbf{u}_{m j}=0$ for $i=N_{l s}, \cdots, N_{s}$. Note that $N_{l s} \geq N_{m}$ and thus one operator may send force commands to more than one slave. The experiment in Section IV shows an example where $N_{m}=3$ and $N_{l s}=4$ and $\tau_{2}$ and $\tau_{3}$ are encodings of a same user command $\mathbf{u}_{m 2}$. Because every $x_{i}$ evolves locally at its corresponding slave, the communication graph of the decision layer is isomorphic to that of the slave network and, thus, it is connected.

Let each decision variable $x_{i}$ evolve according to $\tau_{i}$ and to the states of neighbouring vertices $j^{\prime} \sim i^{\prime}$. Let user $k$ be the winner and send the largest force command $\mathbf{u}_{m k}$ among all users, $\left\|\mathbf{u}_{m k}\right\|>\left\|\mathbf{u}_{m i}\right\|, \forall i=1, \cdots, N_{m}$ and $i \neq k$. Then, the decision-making layer drives the decision variables of all active slaves receiving the winner's command to a positive limit set and reduces the other decision variables to zero. Further, only slaves whose decision variables are above a certain threshold execute their received user commands. Hence, the winner can steer the slave network. For example, let user 1 be 
the winner in Figure 1, i.e., $\left\|\mathbf{u}_{m 1}\right\|>\max \left(\left\|\mathbf{u}_{m 2}\right\|,\left\|\mathbf{u}_{m 3}\right\|\right)$ and thus $\tau_{1}>\tau_{i}$ for $i=2, \cdots, 9$ by (4). Then $x_{1}$ converges to a positive limit set while the other $x_{i}$-s approach zero. In turn, the active slave 1 closes switch 1 and executes $\mathbf{f}_{s 1}=\mathbf{u}_{m 1}$ and the active slaves 2 and 3 open switches 2 and 3 and ignore their received user commands $\mathbf{u}_{m 2}$ and $\mathbf{u}_{m 3}$, i.e., $\mathbf{f}_{s 2}=\mathbf{f}_{s 3}=\mathbf{0}$.

\section{MAIN RESULTS}

To identify the winner, let the evolution law of the decision variable $x_{i}$ of each slave $i$ be:

$$
\dot{x}_{i}=\sigma \cdot\left(N_{s} \tau_{i} x_{i}-\eta_{i} x_{i}\right)
$$

where the subscript $i=1, \cdots, N_{s}$ indexes the vertex $i^{\prime}$ in the decision-making layer, $\tau_{i}$ is given in (4), $\sigma>0$, and:

$$
\eta_{i}=\alpha_{i}+N_{s} \tau_{i} x_{i}^{2}
$$

is a dynamically updated gain with:

$$
\dot{\alpha}_{i}=\sum_{j \sim i} w_{i j}(t) \cdot\left(\eta_{j}-\eta_{i}\right)+h_{i j}(t) \cdot \operatorname{sign}\left(\eta_{j}-\eta_{i}\right)
$$

with $w_{i j}(t)=w_{j i}(t)$ and $h_{i j}(t)=h_{j i}(t)$ to be designed.

Let $M$ be the number of communication links in the slave group. Then, the incidence matrix $\mathbf{D}$ and the unweighted Laplacian matrix $\overline{\mathbf{L}}$ of the decision-making layer network are defined as in [21, Chapter 2]. Let $\mathbf{x}=\left[x_{1}, \cdots, x_{N_{s}}\right]^{\top}$, $\boldsymbol{\tau}=\left[\tau_{1}, \cdots, \tau_{N_{s}}\right]^{\top}$ and $\boldsymbol{\eta}=\left[\eta_{1}, \cdots, \eta_{N_{s}}\right]^{\top}$. Let $" \circ "$ be an operator that takes the product of two vectors componentwisely (their Haddamard product). The $p$-th power of a vector $\mathbf{v}=\left[v_{1}, \cdots, v_{N_{s}}\right]^{\top}$ is thus accordingly $\mathbf{v}^{p}=\left[v_{1}^{p}, \cdots, v_{N_{s}}^{p}\right]^{\top}$. Note that operator " $\circ$ " has higher precedence over operator ". ". The dynamics of the overall decision-making layer then become:

$$
\begin{aligned}
\dot{\mathbf{x}}= & \sigma \cdot\left(N_{s} \boldsymbol{\tau} \circ \mathbf{x}-\boldsymbol{\eta} \circ \mathbf{x}\right), \\
\dot{\boldsymbol{\eta}}= & -\mathbf{L}(t) \cdot \boldsymbol{\eta}-\mathbf{D} \cdot \mathbf{H}(t) \cdot \operatorname{sign}\left(\mathbf{D}^{\top} \cdot \boldsymbol{\eta}\right)+N_{s} \dot{\boldsymbol{\tau}} \circ \mathbf{x}^{2} \\
& +2 \sigma N_{s} \boldsymbol{\tau} \circ \mathbf{x}^{2} \circ\left(N_{s} \boldsymbol{\tau}-\boldsymbol{\eta}\right),
\end{aligned}
$$

where $\mathbf{L}(t)=\mathbf{D} \cdot \mathbf{W}(t) \cdot \mathbf{D}^{\top}$ with $\mathbf{W}(t)=\operatorname{diag}\left\{w_{i j}(t)\right\}$ and $\mathbf{H}(t)=\operatorname{diag}\left\{h_{i j}(t)\right\}$.

In (8), $\boldsymbol{\eta}$ is dynamically updated to estimate $\boldsymbol{\tau}^{\top} \cdot \mathrm{x}^{2} \cdot \mathbf{1}$, where $\mathbf{1}=[1, \cdots, 1]^{\top}$. By (8), the estimating error:

$$
\boldsymbol{\delta}=\boldsymbol{\eta}-\boldsymbol{\tau}^{\top} \cdot \mathbf{x}^{2} \cdot \mathbf{1}
$$

evolves according to:

$$
\begin{aligned}
\dot{\boldsymbol{\delta}}= & \dot{\boldsymbol{\eta}}-\frac{d\left(\boldsymbol{\tau}^{\top} \cdot \mathbf{x}^{2}\right)}{d t} \cdot \mathbf{1} \\
= & -\mathbf{L}(t) \cdot \boldsymbol{\eta}-\mathbf{D} \cdot \mathbf{H}(t) \cdot \operatorname{sign}\left(\mathbf{D}^{\top} \cdot \boldsymbol{\eta}\right)+N_{s} \dot{\boldsymbol{\tau}} \circ \mathbf{x}^{2} \\
& +2 N_{s} \boldsymbol{\tau} \circ \mathbf{x} \circ \dot{\mathbf{x}}-\dot{\boldsymbol{\tau}}^{\top} \cdot \mathbf{x}^{2} \cdot \mathbf{1}-2 \boldsymbol{\tau}^{\top} \cdot(\mathbf{x} \circ \dot{\mathbf{x}}) \cdot \mathbf{1} \\
= & -\mathbf{L}(t) \cdot \boldsymbol{\eta}-\mathbf{D} \cdot \mathbf{H}(t) \cdot \operatorname{sign}\left(\mathbf{D}^{\top} \cdot \boldsymbol{\eta}\right)+\left(N_{s} \mathbf{I}-\mathbf{1} \cdot \mathbf{1}^{\top}\right) \\
& \cdot\left(\dot{\boldsymbol{\tau}} \circ \mathbf{x}^{2}\right)+2 \cdot\left(N_{s} \mathbf{I}-\mathbf{1} \cdot \mathbf{1}^{\top}\right) \cdot(\boldsymbol{\tau} \circ \mathbf{x} \circ \dot{\mathbf{x}}) \\
= & -\mathbf{L}(t) \cdot\left(\boldsymbol{\delta}+\boldsymbol{\tau}^{\top} \cdot \mathbf{x}^{2} \cdot \mathbf{1}\right)-\mathbf{D} \cdot \mathbf{H}(t) \cdot \operatorname{sign}\left[\mathbf{D}^{\top}\right. \\
& \left.\cdot\left(\boldsymbol{\delta}+\boldsymbol{\tau}^{\top} \cdot \mathbf{x}^{2} \cdot \mathbf{1}\right)\right]+\overline{\mathbf{L}}_{c} \cdot\left(\dot{\boldsymbol{\tau}} \circ \mathbf{x}^{2}\right)+2 \overline{\mathbf{L}}_{c} \cdot(\boldsymbol{\tau} \circ \mathbf{x} \circ \dot{\mathbf{x}}) \\
= & -\mathbf{L}(t) \cdot \boldsymbol{\delta}-\mathbf{D} \cdot \mathbf{H}(t) \cdot \operatorname{sign}\left(\mathbf{D}^{\top} \cdot \boldsymbol{\delta}\right)+\overline{\mathbf{L}}_{c} \cdot\left(\dot{\boldsymbol{\tau}} \circ \mathbf{x}^{2}\right) \\
& +2 \sigma \overline{\mathbf{L}}_{c} \cdot\left[\boldsymbol{\tau} \circ \mathbf{x}^{2} \circ\left(N_{s} \boldsymbol{\tau}-\boldsymbol{\eta}\right)\right],
\end{aligned}
$$

where $\overline{\mathbf{L}}_{c}=N_{s} \mathbf{I}-\mathbf{1} \cdot \mathbf{1}^{\top}$ is the unweighted Laplacian of a complete graph of order $N_{s}$.

Let $\bar{\delta}=\mathbf{1}^{\top} \cdot \delta$ be the sum of estimation errors. It follows then that:

Proposition 1. Given $\boldsymbol{\alpha}(0)=\mathbf{0}$, it guarantees that $\bar{\delta}(t)=0$ for all $t \geq 0$.

Proof. Firstly, $\boldsymbol{\alpha}(0)=\mathbf{0}$ leads to

$$
\begin{aligned}
\bar{\delta}(0) & =\mathbf{1}^{\top} \cdot \boldsymbol{\delta}(0)=\mathbf{1}^{\top} \cdot\left[\boldsymbol{\eta}(0)-\boldsymbol{\tau}^{\top}(0) \cdot \mathbf{x}^{2}(0) \cdot \mathbf{1}\right] \\
& =\mathbf{1}^{\top} \cdot \boldsymbol{\eta}(0)-N_{s} \boldsymbol{\tau}^{\top}(0) \cdot \mathbf{x}^{2}(0) \\
& =\mathbf{1}^{\top} \cdot\left[\boldsymbol{\alpha}(0)+N_{s} \boldsymbol{\tau}(0) \circ \mathbf{x}^{2}(0)\right]-N_{s} \boldsymbol{\tau}^{\top}(0) \cdot \mathbf{x}^{2}(0) \\
& =N_{s} \mathbf{1}^{\top} \cdot\left[\boldsymbol{\tau}(0) \circ \mathbf{x}^{2}(0)\right]-N_{s} \boldsymbol{\tau}^{\top}(0) \cdot \mathbf{x}^{2}(0)=0 .
\end{aligned}
$$

Then, $\bar{\delta}$ is invariant by validating its derivative as follows

$$
\begin{aligned}
\dot{\bar{\delta}}= & \mathbf{1}^{\top} \cdot \dot{\boldsymbol{\delta}}=-\mathbf{1}^{\top} \cdot \mathbf{L}(t) \cdot \boldsymbol{\delta}-\mathbf{1}^{\top} \cdot \mathbf{D} \cdot \mathbf{H}(t) \cdot \operatorname{sign}\left(\mathbf{D}^{\top} \cdot \boldsymbol{\delta}\right) \\
& +\mathbf{1}^{\top} \cdot \overline{\mathbf{L}}_{c} \cdot\left(\dot{\boldsymbol{\tau}} \circ \mathbf{x}^{2}\right)+2 \cdot \mathbf{1}^{\top} \cdot \overline{\mathbf{L}}_{c} \cdot(\boldsymbol{\tau} \circ \mathbf{x} \circ \dot{\mathbf{x}})=0,
\end{aligned}
$$

because $\mathbf{L}(t), \overline{\mathbf{L}}_{c}$ are Laplacian matrices and $\mathbf{D}$ is the incidence matrix. Therefore, $\bar{\delta}(t)=\bar{\delta}(0)=0$ for any $t \geq 0$.

The Lyapunov candidate constructed to study the convergence of the decision-making algorithm (5)-(7) is:

$$
V=\frac{1}{4}\left(\mathbf{x}^{\top} \cdot \mathbf{x}-N_{s}\right)^{2}+\frac{\epsilon}{2} \boldsymbol{\delta}^{\top} \cdot \boldsymbol{\delta},
$$

where $\epsilon>0$ will be determined. The first term in (11) measures the deviation from 1 of the decision variables $\boldsymbol{x}$, while the second term quantifies the impact of the estimation error $\boldsymbol{\delta}$.

Define $\boldsymbol{v}=\left[v_{1}, \cdots, v_{M}\right]^{\top}$ with $v_{i}$ the maximum absolute value of all elements in the $i$-th row of $\mathbf{T} \cdot \mathbf{D}^{\top}$. Let $k$ index the oriented link $(i, j)$ defined by the selection of the incidence matrix D [21, Chapter 2]. Further, define $h_{i j}(t)$ by:

$$
h_{i j}(t)=N_{s} \sigma \tilde{\tau} v_{k}\left(\eta_{i}+\eta_{j}\right)
$$

where $\tilde{\tau}=\bar{\tau}-1$ with $\bar{\tau}$ the upper bound of $\tau_{i}$, and select $w_{i j}(t)$ by:

$$
\begin{aligned}
w_{i j}(t)= & {\left[v_{k}^{2}\left(\eta_{i}+\eta_{j}\right)+K_{c} K_{2} M\left(\eta_{i}-\eta_{j}\right)^{2}\right.} \\
& \left.+\frac{K_{c} v_{k}^{4}}{K_{2}}\right] \times\left(2 M \sigma \epsilon \bar{\tau}^{2}+\frac{M \sigma}{4 N_{s}^{2} \epsilon}+\frac{M \sigma}{4 N_{s}}\right) \\
& +\frac{4 M^{2} \tilde{\tau}^{2} v_{k}^{2}}{N_{s} K_{1}}+\frac{N_{s} \sigma^{2} K_{1} v_{k}^{2}}{4}+\varrho K_{c}^{2}
\end{aligned}
$$

where $K_{c}$ is the maximum singular value of $\mathbf{D} \cdot \mathbf{T} / N_{s}$, and $\varrho, K_{1}$ and $K_{2}$ are positive constants.

The derivative of

$$
V_{1}=\frac{1}{4}\left(\mathbf{x}^{\top} \cdot \mathbf{x}-N_{s}\right)^{2}
$$


along Equations (8)-(9) is

$$
\begin{aligned}
\dot{V}_{1}= & \left(\mathbf{x}^{\top} \cdot \mathbf{x}-N_{s}\right) \mathbf{x}^{\top} \cdot \dot{\mathbf{x}} \\
= & \left(\mathbf{x}^{\top} \cdot \mathbf{x}-N_{s}\right) \mathbf{x}^{\top} \cdot \sigma \cdot\left(N_{s} \boldsymbol{\tau} \circ \mathbf{x}-\boldsymbol{\tau}^{\top} \cdot \mathbf{x}^{2} \cdot \mathbf{x}-\boldsymbol{\delta} \circ \mathbf{x}\right) \\
= & \sigma \cdot\left(\mathbf{x}^{\top} \cdot \mathbf{x}-N_{s}\right) \cdot \boldsymbol{\tau}^{\top} \cdot \mathbf{x}^{2} \cdot\left(N_{s}-\mathbf{x}^{\top} \cdot \mathbf{x}\right) \\
& -\sigma \cdot\left(\mathbf{x}^{\top} \cdot \mathbf{x}-N_{s}\right) \cdot \boldsymbol{\delta}^{\top} \cdot \mathbf{x}^{2} \\
\leq & \frac{\sigma}{2} \cdot\left(\mathbf{x}^{\top} \cdot \mathbf{x}-N_{s}\right)^{2} \boldsymbol{\tau}^{\top} \cdot \mathbf{x}^{2}-\sigma \cdot\left(\mathbf{x}^{\top} \cdot \mathbf{x}-N_{s}\right)^{2} \boldsymbol{\tau}^{\top} \cdot \mathbf{x}^{2} \\
& +\frac{\sigma}{2} \cdot(\boldsymbol{\delta} \circ \mathbf{x})^{\top} \cdot \mathbf{U}^{-1} \cdot(\boldsymbol{\delta} \circ \mathbf{x}) \\
= & \frac{\sigma}{2} \cdot(\boldsymbol{\delta} \circ \mathbf{x})^{\top} \cdot \mathbf{U}^{-1} \cdot(\boldsymbol{\delta} \circ \mathbf{x})-\frac{\sigma}{2}\left(\mathbf{x}^{\top} \cdot \mathbf{x}-N_{s}\right)^{2} \boldsymbol{\tau}^{\top} \cdot \mathbf{x}^{2},
\end{aligned}
$$

where $\mathbf{U}=\operatorname{diag}(\mathbf{u})$. Similarly, the derivative of

$$
V_{2}=\frac{1}{2} \boldsymbol{\delta}^{\top} \cdot \boldsymbol{\delta}
$$

along Equations (8)-(10) is

$$
\begin{aligned}
\dot{V}_{2}= & -\boldsymbol{\delta}^{\top} \cdot \mathbf{L}(t) \cdot \boldsymbol{\delta}-\boldsymbol{\delta}^{\top} \cdot \mathbf{D} \cdot \mathbf{H}(t) \cdot \operatorname{sign}\left(\mathbf{D}^{\top} \cdot \boldsymbol{\delta}\right) \\
& +\boldsymbol{\delta}^{\top} \cdot \overline{\mathbf{L}}_{c} \cdot\left(\dot{\boldsymbol{\tau}} \cdot \mathbf{x}^{2}\right)+2 \sigma \boldsymbol{\delta}^{\top} \cdot \overline{\mathbf{L}}_{c} \cdot[\boldsymbol{\tau} \cdot \mathbf{x} \\
& \left.\circ\left(N_{s} \boldsymbol{\tau} \circ \mathbf{x}-\boldsymbol{\tau}^{\top} \mathbf{x}^{2} \cdot \mathbf{x}\right)\right]-2 \sigma \boldsymbol{\delta}^{\top} \cdot \overline{\mathbf{L}}_{c} \cdot\left(\boldsymbol{\tau} \circ \mathbf{x}^{2} \circ \boldsymbol{\delta}\right) \\
= & -\boldsymbol{\delta}^{\top} \cdot \mathbf{L}(t) \cdot \boldsymbol{\delta}-\boldsymbol{\delta}^{\top} \cdot \mathbf{D} \cdot \mathbf{H}(t) \cdot \operatorname{sign}\left(\mathbf{D}^{\top} \cdot \boldsymbol{\delta}\right) \\
& +\boldsymbol{\delta}^{\top} \cdot \overline{\mathbf{L}}_{c} \cdot\left(\dot{\boldsymbol{\tau}} \circ \mathbf{x}^{2}\right)+2 \sigma \boldsymbol{\delta} \cdot \overline{\mathbf{L}}_{c} \cdot\left(\boldsymbol { \tau } \circ \mathbf { x } \circ \left[\left(N_{s} \mathbf{I}\right.\right.\right. \\
& \left.\left.\left.-\mathbf{x} \cdot \mathbf{x}^{\top}\right) \cdot(\boldsymbol{\tau} \circ \mathbf{x})\right]\right)-2 N_{s} \sigma \boldsymbol{\delta}^{\top} \cdot \mathbf{U} \cdot \mathbf{X} \cdot \mathbf{X} \cdot \boldsymbol{\delta},
\end{aligned}
$$

where $\overline{\mathbf{L}}_{c} \cdot \boldsymbol{\delta}=N_{s} \boldsymbol{\delta}$ by Proposition 1 , and $\mathbf{X}=\operatorname{diag}(\mathbf{x})$.

Notice that $\tau \circ \mathrm{x}$ can be geometrically decomposed into $\tau \circ \mathbf{x}=\mathrm{x}^{\|}+\mathrm{x}^{\perp}$, where

$$
\mathbf{x}^{\|}=\frac{\mathbf{x} \cdot \mathbf{x}^{\top}}{\|\mathbf{x}\|^{2}}(\boldsymbol{\tau} \circ \mathbf{x}), \quad \text { and } \quad \mathbf{x}^{\perp}=\left(\mathbf{I}-\frac{\mathbf{x} \cdot \mathbf{x}^{\boldsymbol{\top}}}{\|\mathbf{x}\|^{2}}\right) \cdot(\boldsymbol{\tau} \circ \mathbf{x}) .
$$

It then follows that

$$
\begin{aligned}
& \left(N_{s} \mathbf{I}-\mathbf{x} \cdot \mathbf{x}^{\boldsymbol{\top}}\right) \mathbf{x}^{\|}=\left(N_{s} \mathbf{I}-\mathbf{x} \cdot \mathbf{x}^{\boldsymbol{\top}}\right) \frac{\mathbf{x} \cdot \mathbf{x}^{\top}}{\|\mathbf{x}\|^{2}}(\boldsymbol{\tau} \circ \mathbf{x}) \\
= & \frac{\mathbf{x}^{\top} \cdot \mathbf{U} \cdot \mathbf{x}}{\|\mathbf{x}\|^{2}}\left(N_{s}-\mathbf{x}^{\top} \cdot \mathbf{x}\right) \mathbf{x}
\end{aligned}
$$

and that

$$
\begin{aligned}
& \left(N_{s} \mathbf{I}-\mathbf{x} \cdot \mathbf{x}^{\top}\right) \mathbf{x}^{\perp} \\
= & \left(N_{s} \mathbf{I}-\mathbf{x} \cdot \mathbf{x}^{\top}\right) \cdot\left(\mathbf{I}-\frac{\mathbf{x} \cdot \mathbf{x}^{\top}}{\|\mathbf{x}\|^{2}}\right) \cdot(\boldsymbol{\tau} \circ \mathbf{x}) \\
= & \left(N_{s} \mathbf{I}-N_{s} \frac{\mathbf{x} \cdot \mathbf{x}^{\top}}{\|\mathbf{x}\|^{2}}-\mathbf{x} \cdot \mathbf{x}^{\top}+\frac{\mathbf{x} \cdot \mathbf{x}^{\top} \cdot \mathbf{x} \cdot \mathbf{x}^{\top}}{\|\mathbf{x}\|^{2}}\right) \cdot(\boldsymbol{\tau} \circ \mathbf{x}) \\
= & N_{s} \cdot\left(\mathbf{I}-\frac{\mathbf{x} \cdot \mathbf{x}^{\top}}{\|\mathbf{x}\|^{2}}\right) \cdot(\boldsymbol{\tau} \circ \mathbf{x})=N_{s} \mathbf{x}^{\perp} .
\end{aligned}
$$

Further, we have

$$
\begin{aligned}
& 2 \sigma \boldsymbol{\delta}^{\top} \cdot \overline{\mathbf{L}}_{c} \cdot\left(\boldsymbol{\tau} \circ \mathbf{x} \circ\left[\left(N_{s} \mathbf{I}-\mathbf{x} \cdot \mathbf{x}^{\top}\right) \mathbf{x}^{\|}\right]\right) \\
= & 2 \sigma \boldsymbol{\delta}^{\top} \cdot \overline{\mathbf{L}}_{c} \cdot\left[(\boldsymbol{\tau} \circ \mathbf{x}) \circ \frac{\mathbf{x}^{\boldsymbol{\top}} \cdot \mathbf{U} \cdot \mathbf{x}}{\|\mathbf{x}\|^{2}}\left(N_{s}-\mathbf{x}^{\boldsymbol{\top}} \cdot \mathbf{x}\right) \mathbf{x}\right] \\
\leq & \frac{\sigma}{4 \epsilon}\left(\mathbf{x}^{\top} \cdot \mathbf{x}-N_{s}\right)^{2} \boldsymbol{\tau}^{\top} \cdot \mathbf{x}^{2}+4 \sigma \epsilon \bar{\tau}^{2} \boldsymbol{\delta}^{\top} \cdot \overline{\mathbf{L}}_{c} \cdot \mathbf{U} \cdot \mathbf{X} \cdot \mathbf{X} \cdot \overline{\mathbf{L}}_{c} \cdot \boldsymbol{\delta} .
\end{aligned}
$$

Because $\overline{\mathbf{L}}_{c}$ can be decomposed by a positive semi-definite $\mathbf{T}$ into $\overline{\mathbf{L}}_{c}=\mathbf{D} \cdot \mathbf{T} \cdot \mathbf{D}^{\top}$, it follows that

$$
\begin{aligned}
& 2 \sigma \boldsymbol{\delta}^{\top} \cdot \overline{\mathbf{L}}_{c} \cdot\left(\boldsymbol{\tau} \circ \mathbf{x} \circ\left[\left(N_{s} \mathbf{I}-\mathbf{x} \cdot \mathbf{x}^{\top}\right) \mathbf{x}^{\perp}\right]\right) \\
= & 2 N_{s} \sigma \boldsymbol{\delta}^{\top} \cdot \overline{\mathbf{L}}_{c} \cdot\left(\boldsymbol{\tau} \circ \mathbf{x} \circ \mathbf{x}^{\perp}\right) \\
= & 2 N_{s} \sigma \boldsymbol{\delta}^{\top} \cdot \mathbf{D} \cdot \mathbf{T} \cdot \mathbf{D}^{\top} \cdot\left(\boldsymbol{\tau} \circ \mathbf{x} \circ \mathbf{x}^{\perp}\right) \\
\leq & 2 N_{s} \sigma|\tilde{\boldsymbol{\delta}}|^{\top} \cdot\left|\mathbf{T} \cdot \mathbf{D}^{\top} \cdot\left(\boldsymbol{\tau} \circ \mathbf{x} \circ \mathbf{x}^{\perp}\right)\right| \\
= & 2 N_{s} \sigma|\tilde{\boldsymbol{\delta}}|^{\top} \cdot\left|\mathbf{T} \cdot \mathbf{D}^{\top} \cdot\left(\boldsymbol{\tau} \circ \mathbf{x} \circ\left[\left(\mathbf{I}-\frac{\mathbf{x} \cdot \mathbf{x}^{\top}}{\|\mathbf{x}\|^{2}}\right) \cdot(\boldsymbol{\tau} \circ \mathbf{x})\right]\right)\right| \\
= & 2 N_{s} \sigma|\tilde{\boldsymbol{\delta}}|^{\top} \cdot\left|\mathbf{T} \cdot \mathbf{D}^{\top} \cdot\left[\left(\boldsymbol{\tau}-\frac{\boldsymbol{\tau}^{\top} \cdot \mathbf{x}^{2}}{\|\mathbf{x}\|^{2}} \mathbf{1}\right) \circ \boldsymbol{\tau} \circ \mathbf{x}^{2}\right]\right| \\
\leq & 2 N_{s} \sigma|\tilde{\boldsymbol{\delta}}|^{\top} \cdot\left|\boldsymbol{v} \cdot \mathbf{1}^{\top} \cdot\left(\tilde{\tau} \boldsymbol{\tau} \circ \mathbf{x}^{2}\right)\right| \\
= & 2 N_{s} \sigma \tilde{\tau} \cdot\left(\boldsymbol{\tau}^{\top} \cdot \mathbf{x}^{2}\right) \cdot|\tilde{\boldsymbol{\delta}}|^{\top} \cdot \boldsymbol{v} \\
= & N_{s} \sigma \tilde{\tau} \cdot|\tilde{\boldsymbol{\delta}}|^{\top} \cdot\left(\boldsymbol{v} \circ\left[|\mathbf{D}|^{\top} \cdot(\boldsymbol{\eta}-\boldsymbol{\delta})\right]\right) \\
\leq & N_{s} \sigma \tilde{\tau}|\tilde{\boldsymbol{\delta}}|^{\top} \cdot \boldsymbol{\Upsilon} \cdot|\mathbf{D}|^{\top} \cdot \boldsymbol{\eta}+\frac{N_{s} \sigma^{2} K_{1}}{4} \tilde{\boldsymbol{\delta}} \cdot \boldsymbol{\Upsilon}^{2} \cdot \tilde{\boldsymbol{\delta}} \\
& +\frac{N_{s} \tilde{\tau}^{2}}{K_{1}} \cdot \boldsymbol{\delta}^{\top} \cdot|\mathbf{D}| \cdot|\mathbf{D}|^{\top} \cdot \boldsymbol{\delta},
\end{aligned}
$$

where $\tilde{\boldsymbol{\delta}}=\mathbf{D}^{\top} \cdot \boldsymbol{\delta}, \boldsymbol{\Upsilon}=\operatorname{diag}(\boldsymbol{v}), \tau_{i} x_{i}^{2}>0$ and

$$
\left|\tau_{i}-\frac{\boldsymbol{\tau}^{\top} \cdot \mathbf{x}^{2}}{\|\mathbf{x}\|^{2}}\right| \leq \tilde{\tau}
$$

have been employed to obtain the seventh line, and $2 \tau^{\top} \cdot \mathrm{x}^{2} \cdot 1$ is replaced with $|\mathbf{D}|^{\top} \cdot(\boldsymbol{\eta}-\boldsymbol{\delta})$ to get the ninth line.

Because $\tau \circ \mathbf{x}=\mathbf{x}^{\|}+\mathbf{x}^{\perp}$, the summation of the above two equations indicates that

$$
\begin{aligned}
& 2 \sigma \boldsymbol{\delta}^{\top} \cdot \overline{\mathbf{L}}_{c} \cdot\left(\boldsymbol{\tau} \circ \mathbf{x} \circ\left[\left(N_{s} \mathbf{I}-\mathbf{x} \cdot \mathbf{x}^{\top}\right) \cdot(\boldsymbol{\tau} \circ \mathbf{x})\right]\right) \\
\leq & \frac{\sigma}{4 \epsilon} \cdot\left(\mathbf{x}^{\top} \cdot \mathbf{x}-N_{s}\right)^{2} \boldsymbol{\tau}^{\top} \cdot \mathbf{x}^{2}+N_{s} \sigma \tilde{\tau} \cdot|\tilde{\boldsymbol{\delta}}|^{\top} \cdot \boldsymbol{\Upsilon} \cdot|\mathbf{D}|^{\top} \cdot \boldsymbol{\eta} \\
& +\frac{N_{s} \sigma^{2} K_{1}}{4} \tilde{\boldsymbol{\delta}}^{\top} \cdot \boldsymbol{\Upsilon} \cdot \boldsymbol{\Upsilon} \cdot \tilde{\boldsymbol{\delta}} \\
& +\boldsymbol{\delta}^{\boldsymbol{\top}} \cdot\left(4 N_{s}^{2} \sigma \epsilon \bar{\tau}^{2} \mathbf{U} \cdot \mathbf{X} \cdot \mathbf{X}+\frac{N_{s} \tilde{\tau}^{2}}{K_{1}}|\mathbf{D}| \cdot|\mathbf{D}|^{\top}\right) \cdot \boldsymbol{\delta} .
\end{aligned}
$$

Simple algebraic operations lead to

$$
\begin{aligned}
& \boldsymbol{\delta}^{\boldsymbol{\top}} \cdot \overline{\mathbf{L}}_{c} \cdot\left(\dot{\boldsymbol{\tau}} \circ \mathbf{x}^{2}\right)=N_{s} \boldsymbol{\delta}^{\boldsymbol{\top}} \cdot \mathbf{X}^{2} \cdot \dot{\boldsymbol{\tau}} \\
\leq & 2 N_{s} \sigma \boldsymbol{\delta}^{\boldsymbol{\top}} \cdot \mathbf{U} \cdot \mathbf{X} \cdot \mathbf{X} \cdot \boldsymbol{\delta}+\frac{N_{s}}{8 \sigma} \dot{\boldsymbol{\tau}}^{\boldsymbol{\top}} \cdot \mathbf{U}^{-1} \cdot \mathbf{X} \cdot \mathbf{X} \cdot \dot{\boldsymbol{\tau}}
\end{aligned}
$$

By the above two equations, $\dot{V}_{2}$ becomes

$$
\begin{aligned}
\dot{V}_{2} \leq & -\boldsymbol{\delta}^{\boldsymbol{\top}} \cdot \mathbf{L}(t) \cdot \boldsymbol{\delta}-\tilde{\boldsymbol{\delta}} \cdot \mathbf{H}(t) \cdot \operatorname{sign}(\tilde{\boldsymbol{\delta}}) \\
& +\frac{N_{s}}{8 \sigma} \dot{\boldsymbol{\tau}}^{\boldsymbol{\top}} \cdot \mathbf{U}^{-1} \cdot \mathbf{X} \cdot \mathbf{X} \cdot \dot{\boldsymbol{\tau}}+\frac{\sigma}{4 \epsilon}\left(\mathbf{x}^{\boldsymbol{\top}} \cdot \mathbf{x}-N_{s}\right)^{2} \boldsymbol{\tau}^{\boldsymbol{\top}} \cdot \mathbf{x}^{2} \\
& +N_{s} \sigma \tilde{\tau}|\tilde{\boldsymbol{\delta}}|^{\top} \cdot \boldsymbol{\Upsilon} \cdot|\mathbf{D}|^{\top} \cdot \boldsymbol{\eta}+\frac{N_{s} \sigma^{2} K_{1}}{4} \tilde{\boldsymbol{\delta}} \cdot \boldsymbol{\Upsilon} \cdot \boldsymbol{\Upsilon} \cdot \tilde{\boldsymbol{\delta}} \\
& +\boldsymbol{\delta}^{\boldsymbol{\top}} \cdot\left(4 N_{s}^{2} \sigma \epsilon \bar{\tau}^{2} \mathbf{U} \cdot \mathbf{X} \cdot \mathbf{X}+\frac{N_{s} \tilde{\tau}^{2}}{K_{1}}|\mathbf{D}| \cdot|\mathbf{D}|^{\top}\right) \cdot \boldsymbol{\delta} .
\end{aligned}
$$

The selection of $h_{i j}(t)$ in (12) for every $i \sim j$ makes that

$$
-\tilde{\boldsymbol{\delta}}^{\top} \cdot \mathbf{H}(t) \cdot \operatorname{sign}(\tilde{\boldsymbol{\delta}})+N_{s} \sigma \tilde{\tau}|\tilde{\boldsymbol{\delta}}|^{\top} \cdot \boldsymbol{\Upsilon} \cdot|\mathbf{D}|^{\top} \cdot \boldsymbol{\eta}=0 .
$$


Thus, combining $\dot{V}_{1}$ and $\dot{V}_{2}$ and using the above upper-bound $\dot{V}=\dot{V}_{1}+\epsilon \dot{V}_{2}$ by

$$
\begin{aligned}
& \dot{V} \leq-\frac{\sigma}{2} \cdot\left(\mathbf{x}^{\top} \cdot \mathbf{x}-N_{s}\right)^{2} \boldsymbol{\tau}^{\top} \cdot \mathbf{x}^{2}+\frac{\sigma}{2} \boldsymbol{\delta}^{\top} \cdot \mathbf{U}^{-1} \cdot \mathbf{X} \cdot \mathbf{X} \cdot \boldsymbol{\delta} \\
& +\frac{N_{s} \sigma^{2} \epsilon K_{1}}{4} \tilde{\boldsymbol{\delta}}^{\boldsymbol{\top}} \cdot \mathbf{\Upsilon} \cdot \boldsymbol{\Upsilon} \cdot \tilde{\boldsymbol{\delta}}+\frac{\sigma}{4} \cdot\left(\mathbf{x}^{\boldsymbol{\top}} \cdot \mathbf{x}-N_{s}\right)^{2} \boldsymbol{\tau}^{\boldsymbol{\top}} \cdot \mathbf{x}^{2} \\
& +\boldsymbol{\delta}^{\top} \cdot\left(4 N_{s}^{2} \sigma \epsilon^{2} \bar{\tau}^{2} \mathbf{U} \cdot \mathbf{X} \cdot \mathbf{X}+\frac{N_{s} \epsilon \tilde{\tau}^{2}}{K_{1}}|\mathbf{D}| \cdot|\mathbf{D}|^{\top}\right) \cdot \boldsymbol{\delta} \\
& +\frac{N_{s} \epsilon}{8 \sigma} \dot{\boldsymbol{\tau}}^{\top} \cdot \mathbf{U}^{-1} \cdot \mathbf{X} \cdot \mathbf{X} \cdot \dot{\boldsymbol{\tau}}-\epsilon \cdot \boldsymbol{\delta}^{\top} \cdot \mathbf{L}(t) \cdot \boldsymbol{\delta} \\
& =-\frac{\sigma}{4} \cdot\left(\mathbf{x}^{\top} \cdot \mathbf{x}-N_{s}\right)^{2} \boldsymbol{\tau}^{\top} \cdot \mathbf{x}^{2}-\frac{\sigma \epsilon}{2} \cdot \boldsymbol{\delta}^{\top} \cdot \boldsymbol{\delta} \cdot \boldsymbol{\tau}^{\top} \cdot \mathbf{x}^{2} \\
& +\frac{N_{s} \epsilon}{8 \sigma} \dot{\boldsymbol{\tau}}^{\top} \cdot \mathbf{U}^{-1} \cdot \mathbf{X} \cdot \mathbf{X} \cdot \dot{\boldsymbol{\tau}}-\epsilon \cdot \boldsymbol{\delta}^{\top} \cdot \mathbf{L}(t) \cdot \boldsymbol{\delta} \\
& +\epsilon \cdot \boldsymbol{\delta}^{\top} \cdot \boldsymbol{\Lambda}(t) \cdot \boldsymbol{\delta}+\frac{N_{s} \sigma^{2} \epsilon K_{1}}{4} \tilde{\boldsymbol{\delta}}^{\top} \cdot \boldsymbol{\Upsilon} \cdot \boldsymbol{\Upsilon} \cdot \tilde{\boldsymbol{\delta}},
\end{aligned}
$$

where $(\sigma \epsilon / 2) \cdot \boldsymbol{\delta}^{\top} \cdot \boldsymbol{\delta} \cdot \boldsymbol{\tau}^{\top} \cdot \mathrm{x}^{2}$ has been added and subtracted in the above with

$$
\begin{aligned}
\boldsymbol{\Lambda}(t)= & 4 N_{s}^{2} \sigma \epsilon \bar{\tau}^{2} \mathbf{U} \cdot \mathbf{X} \cdot \mathbf{X}+\frac{\sigma}{2 \epsilon} \mathbf{U}^{-1} \cdot \mathbf{X} \cdot \mathbf{X} \\
& +\frac{\sigma}{2} \boldsymbol{\tau}^{\top} \cdot \mathbf{X}^{2} \cdot \mathbf{I}+\frac{N_{s} \tilde{\tau}^{2}}{K_{1}}|\mathbf{D}| \cdot|\mathbf{D}|^{\top}
\end{aligned}
$$

Proposition 2. The error of estimation $\delta$ can be upperbounded by the difference $\tilde{\boldsymbol{\delta}}$ between the estimating errors as below:

$$
\boldsymbol{\delta}^{\top} \cdot \boldsymbol{\delta} \leq K_{c}^{2} \tilde{\boldsymbol{\delta}}^{\top} \cdot \tilde{\boldsymbol{\delta}}
$$

Proof. Because $\overline{\mathbf{L}}_{c} \cdot \boldsymbol{\delta}=\left(N_{s} \mathbf{I}-\mathbf{1} \cdot \mathbf{1}^{\boldsymbol{\top}}\right) \cdot \boldsymbol{\delta}=N_{s} \boldsymbol{\delta}$,

$$
\begin{aligned}
\boldsymbol{\delta}^{\top} \cdot \boldsymbol{\delta} & =\frac{1}{N_{s}^{2}} \boldsymbol{\delta}^{\top} \cdot \overline{\mathbf{L}}_{c} \cdot \overline{\mathbf{L}}_{c} \cdot \boldsymbol{\delta} \\
& =\frac{1}{N_{s}^{2}} \boldsymbol{\delta}^{\top} \cdot \mathbf{D} \cdot \mathbf{T} \cdot \mathbf{D}^{\top} \cdot \mathbf{D} \cdot \mathbf{T} \cdot \mathbf{D}^{\top} \cdot \boldsymbol{\delta} \\
& =\frac{1}{N_{s}^{2}} \tilde{\boldsymbol{\delta}}^{\top} \cdot \mathbf{T} \cdot \mathbf{D}^{\top} \cdot \mathbf{D} \cdot \mathbf{T} \cdot \tilde{\boldsymbol{\delta}} \leq K_{c}^{2} \tilde{\boldsymbol{\delta}}^{\top} \cdot \tilde{\boldsymbol{\delta}}
\end{aligned}
$$

Notice firstly that

$$
\begin{aligned}
& \boldsymbol{\delta}^{\top} \cdot \boldsymbol{\Lambda}(t) \cdot \boldsymbol{\delta}=\frac{1}{N_{s}^{2}} \boldsymbol{\delta}^{\top} \cdot \overline{\mathbf{L}}_{c} \cdot \mathbf{\Lambda}(t) \cdot \overline{\mathbf{L}}_{c} \cdot \boldsymbol{\delta} \\
= & \frac{1}{N_{s}^{2}} \boldsymbol{\delta}^{\top} \cdot \mathbf{D} \cdot \mathbf{T} \cdot \mathbf{D}^{\top} \cdot \boldsymbol{\Lambda}(t) \cdot \mathbf{D} \cdot \mathbf{T} \cdot \mathbf{D}^{\top} \cdot \boldsymbol{\delta} \\
= & \frac{1}{N_{s}^{2}} \tilde{\boldsymbol{\delta}}^{\top} \cdot \mathbf{T} \cdot \mathbf{D}^{\top} \cdot \mathbf{\Lambda}(t) \cdot \mathbf{D} \cdot \mathbf{T} \cdot \tilde{\boldsymbol{\delta}} \\
\leq & \frac{1}{N_{s}^{2}}|\tilde{\boldsymbol{\delta}}|^{\top} \cdot \boldsymbol{v} \cdot \mathbf{1}^{\top} \cdot \mathbf{\Lambda}(t) \cdot \mathbf{1} \cdot \boldsymbol{v}^{\top} \cdot|\tilde{\boldsymbol{\delta}}| \\
= & {\left[4 \sigma \epsilon \bar{\tau}^{2} \mathbf{1}^{\top} \cdot \mathbf{U} \cdot \mathbf{X} \cdot \mathbf{X} \cdot \mathbf{1}+\frac{\sigma}{2 N_{s}^{2} \epsilon} \mathbf{1}^{\top} \cdot \mathbf{U}^{-1} \cdot \mathbf{X} \cdot \mathbf{X} \cdot \mathbf{1}\right.} \\
& \left.+\frac{\sigma}{2 N_{s}} \boldsymbol{\tau}^{\top} \cdot \mathbf{x}^{2}+\frac{\tilde{\tau}^{2}}{N_{s} K_{1}} \mathbf{1}^{\top} \cdot|\mathbf{D}| \cdot|\mathbf{D}|^{\top} \cdot \mathbf{1}\right] \cdot\left(\boldsymbol{v}^{\top} \cdot \tilde{\boldsymbol{\delta}}\right)^{2} \\
= & \left(4 \sigma \epsilon \bar{\tau}^{2} \boldsymbol{\tau}^{\top} \cdot \mathbf{x}^{2}+\frac{\sigma}{2 N_{s}^{2} \epsilon} \mathbf{x}^{\top} \cdot \mathbf{U}^{-1} \cdot \mathbf{x}\right. \\
& \left.+\frac{\sigma}{2 N_{s}} \boldsymbol{\tau}^{\top} \cdot \mathbf{x}^{2}+\frac{4 M \tilde{\tau}^{2}}{N_{s} K_{1}}\right) \cdot\left(\boldsymbol{v}^{\top} \cdot \tilde{\boldsymbol{\delta}}\right)^{2}
\end{aligned}
$$

$$
\begin{aligned}
\leq & \left(4 M \sigma \epsilon \bar{\tau}^{2} \boldsymbol{\tau}^{\top} \cdot \mathbf{x}^{2}+\frac{M \sigma}{2 N_{s}^{2} \epsilon} \mathbf{x}^{\top} \cdot \mathbf{U}^{-1} \cdot \mathbf{x}\right. \\
& \left.+\frac{M \sigma}{2 N_{s}} \boldsymbol{\tau}^{\top} \cdot \mathbf{x}^{2}+\frac{4 M \tilde{\tau}^{2}}{N_{s} K_{1}}\right) \cdot \tilde{\boldsymbol{\delta}}^{\top} \cdot \boldsymbol{\Upsilon} \cdot \boldsymbol{\Upsilon} \cdot \tilde{\boldsymbol{\delta}} \\
\leq & {\left[\left(2 M \sigma \epsilon \bar{\tau}^{2}+\frac{M \sigma}{4 N_{s}^{2} \epsilon}+\frac{M \sigma}{4 N_{s}}\right) \cdot 2 \boldsymbol{\tau}^{\top} \cdot \mathbf{x}^{2}\right.} \\
& \left.+\frac{4 M^{2} \tilde{\tau}^{2}}{N_{s} K_{1}}\right] \cdot \tilde{\boldsymbol{\delta}}^{\top} \cdot \boldsymbol{\Upsilon} \cdot \boldsymbol{\Upsilon} \cdot \tilde{\boldsymbol{\delta}} .
\end{aligned}
$$

Rewritting $2 \boldsymbol{\tau}^{\top} \cdot \mathbf{x}^{2} \cdot \tilde{\boldsymbol{\delta}}^{\top} \cdot \boldsymbol{\Upsilon} \cdot \boldsymbol{\Upsilon} \cdot \tilde{\boldsymbol{\delta}}$ in the above by

$$
\begin{aligned}
& 2 \boldsymbol{\tau}^{\top} \cdot \mathbf{x}^{2} \cdot \tilde{\boldsymbol{\delta}}^{\top} \cdot \boldsymbol{\Upsilon} \cdot \boldsymbol{\Upsilon} \cdot \tilde{\boldsymbol{\delta}} \\
= & \tilde{\boldsymbol{\delta}}^{\boldsymbol{T}} \cdot \boldsymbol{\Upsilon} \cdot \boldsymbol{\Upsilon} \cdot \operatorname{diag}\left\{|\mathbf{D}|^{\top} \cdot(\boldsymbol{\eta}-\boldsymbol{\delta})\right\} \cdot \tilde{\boldsymbol{\delta}}^{\top} \\
= & \tilde{\boldsymbol{\delta}}^{\top} \cdot \boldsymbol{\Upsilon} \cdot \boldsymbol{\Upsilon} \cdot \operatorname{diag}\left\{|\mathbf{D}|^{\top} \cdot \boldsymbol{\eta}\right\} \cdot \tilde{\boldsymbol{\delta}} \\
& -\tilde{\boldsymbol{\delta}}^{\boldsymbol{\top}} \cdot \boldsymbol{\Upsilon} \cdot \boldsymbol{\Upsilon} \cdot \operatorname{diag}\left\{|\mathbf{D}|^{\top} \cdot \boldsymbol{\delta}\right\} \cdot \tilde{\boldsymbol{\delta}},
\end{aligned}
$$

where the last term can be upper-bounded by Proposition 2

$$
\begin{aligned}
& -\tilde{\boldsymbol{\delta}}^{\top} \cdot \boldsymbol{\Upsilon} \cdot \boldsymbol{\Upsilon} \cdot \operatorname{diag}\left\{|\mathbf{D}|^{\top} \cdot \boldsymbol{\delta}\right\} \cdot \tilde{\boldsymbol{\delta}} \\
& \leq 2|\boldsymbol{\delta}|_{\infty} \cdot \tilde{\boldsymbol{\delta}}^{\top} \cdot \boldsymbol{\Upsilon} \cdot \boldsymbol{\Upsilon} \cdot \tilde{\boldsymbol{\delta}}
\end{aligned}
$$

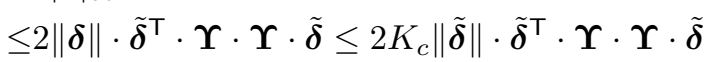

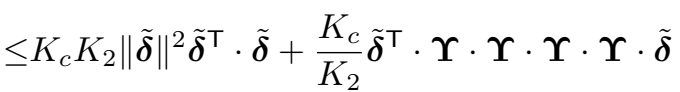

$$
\begin{aligned}
& \leq K_{c} K_{2} M \tilde{\boldsymbol{\delta}}^{\top} \cdot \operatorname{diag}\left\{\left(\mathbf{D}^{\top} \cdot \boldsymbol{\eta}\right)^{2}\right\} \cdot \tilde{\boldsymbol{\delta}}^{\top} \\
& +\frac{K_{c}}{K_{2}} \tilde{\boldsymbol{\delta}}^{\top} \cdot \boldsymbol{\Upsilon} \cdot \boldsymbol{\Upsilon} \cdot \boldsymbol{\Upsilon} \cdot \boldsymbol{\Upsilon} \cdot \tilde{\boldsymbol{\delta}} .
\end{aligned}
$$

Together, we have

$$
\boldsymbol{\delta}^{\top} \cdot \boldsymbol{\Lambda}(t) \cdot \boldsymbol{\delta} \leq \tilde{\boldsymbol{\delta}}^{\top} \cdot \boldsymbol{\Gamma}(t) \cdot \tilde{\boldsymbol{\delta}}
$$

where $\boldsymbol{\Gamma}(t)$ is given by

$$
\begin{aligned}
\boldsymbol{\Gamma}(t)= & \left(2 M \sigma \epsilon \bar{\tau}^{2}+\frac{M \sigma}{4 N_{s}^{2} \epsilon}+\frac{M \sigma}{4 N_{s}}\right) \cdot\left(\frac{K_{c}}{K_{2}} \boldsymbol{\Upsilon} \cdot \boldsymbol{\Upsilon} \cdot \boldsymbol{\Upsilon} \cdot \boldsymbol{\Upsilon}\right. \\
& +K_{c} K_{2} M \cdot \operatorname{diag}\left\{\left(\mathbf{D}^{\top} \cdot \boldsymbol{\eta}\right)^{2}\right\} \\
& \left.+\boldsymbol{\Upsilon} \cdot \boldsymbol{\Upsilon} \cdot \operatorname{diag}\left\{|\mathbf{D}|^{\top} \cdot \boldsymbol{\eta}\right\}\right)+\frac{4 M^{2} \tilde{\tau}^{2}}{N_{s} K_{1}} \boldsymbol{\Upsilon} \cdot \boldsymbol{\Upsilon}
\end{aligned}
$$

And $\dot{V}$ thus becomes

$$
\begin{aligned}
& \dot{V} \leq-\frac{\sigma}{4} \cdot\left(\mathbf{x}^{\top} \cdot \mathbf{x}-N_{s}\right)^{2} \boldsymbol{\tau}^{\top} \cdot \mathbf{x}^{2}-\frac{\sigma \epsilon}{2} \boldsymbol{\delta}^{\top} \cdot \boldsymbol{\delta} \cdot \boldsymbol{\tau}^{\top} \cdot \mathbf{x}^{2} \\
& +\frac{N_{s} \epsilon}{8 \sigma} \dot{\boldsymbol{\tau}}^{\top} \cdot \mathbf{U}^{-1} \cdot \mathbf{X} \cdot \mathbf{X} \cdot \dot{\boldsymbol{\tau}}-\epsilon \boldsymbol{\delta}^{\top} \cdot \mathbf{L}(t) \cdot \boldsymbol{\delta} \\
& +\epsilon \tilde{\boldsymbol{\delta}}^{\top} \cdot \boldsymbol{\Gamma}(t) \cdot \tilde{\boldsymbol{\delta}}+\frac{N_{s} \sigma^{2} \epsilon K_{1}}{4} \tilde{\boldsymbol{\delta}}^{\top} \cdot \mathbf{\Upsilon} \cdot \boldsymbol{\Upsilon} \cdot \tilde{\boldsymbol{\delta}} \\
& \leq-\frac{\sigma}{4} \cdot\left(\mathbf{x}^{\top} \cdot \mathbf{x}-N_{s}\right)^{2} \boldsymbol{\tau}^{\top} \cdot \mathbf{x}^{2}-\frac{\sigma \epsilon}{2} \boldsymbol{\delta}^{\top} \cdot \boldsymbol{\delta} \cdot \boldsymbol{\tau}^{\top} \cdot \mathbf{x}^{2} \\
& +\frac{N_{s} \epsilon\|\dot{\boldsymbol{\tau}}\|^{2}}{8 \sigma} \boldsymbol{\tau}^{\top} \cdot \mathbf{x}^{2}-\epsilon \boldsymbol{\delta}^{\top} \cdot \mathbf{D} \cdot \mathbf{W}(t) \cdot \mathbf{D}^{\top} \cdot \boldsymbol{\delta} \\
& +\epsilon \tilde{\boldsymbol{\delta}}^{\boldsymbol{T}} \cdot \boldsymbol{\Gamma}(t) \cdot \tilde{\boldsymbol{\delta}}+\frac{N_{s} \sigma^{2} \epsilon K_{1}}{4} \tilde{\boldsymbol{\delta}}^{\boldsymbol{\top}} \cdot \boldsymbol{\Upsilon} \cdot \boldsymbol{\Upsilon} \cdot \tilde{\boldsymbol{\delta}} \\
& =-\frac{\sigma}{4} \cdot\left(\mathbf{x}^{\top} \cdot \mathbf{x}-N_{s}\right)^{2} \boldsymbol{\tau}^{\top} \cdot \mathbf{x}^{2} \\
& -\frac{\sigma \epsilon}{2} \boldsymbol{\delta}^{\top} \cdot \boldsymbol{\delta} \cdot \boldsymbol{\tau}^{\top} \cdot \mathbf{x}^{2}+\frac{N_{s} \epsilon\|\dot{\boldsymbol{\tau}}\|^{2}}{8 \sigma} \boldsymbol{\tau}^{\top} \cdot \mathbf{x}^{2} \\
& -\epsilon \tilde{\boldsymbol{\delta}}^{\boldsymbol{T}} \cdot\left[\mathbf{W}(t)-\boldsymbol{\Gamma}(t)-\frac{N_{s} \sigma^{2} K_{1}}{4} \boldsymbol{\Upsilon} \cdot \boldsymbol{\Upsilon}\right] \cdot \tilde{\boldsymbol{\delta}} .
\end{aligned}
$$


Choose $w_{i j}(t)$ by (13) for every $i \sim j$. The derivative of $V$ can then be upper-bounded by:

$$
\dot{V} \leq-\kappa(t) \cdot V-\epsilon \varrho \boldsymbol{\delta}^{\top} \cdot \boldsymbol{\delta}+\kappa(t) \cdot \chi\left(\|\dot{\boldsymbol{\tau}}(t)\|^{2}\right),
$$

where the dependence of $\kappa(t)$ and $\chi\left(\|\dot{\boldsymbol{\tau}}(t)\|^{2}\right)$ on time is highlighted for the ease of obtaining (15), and:

$$
\kappa(t)=\sigma \boldsymbol{\tau}^{\top} \cdot \mathbf{x}^{2} \quad \text { and } \quad \chi\left(\|\dot{\boldsymbol{\tau}}(t)\|^{2}\right)=\frac{N \epsilon\|\dot{\boldsymbol{\tau}}\|^{2}}{8 \sigma^{2}} .
$$

Time integration of (14) from $t_{0} \geq 0$ to $t \geq t_{0}$ yields:

$$
V(t) \leq \exp \left(-\int_{t_{0}}^{t} \kappa(\theta) d \theta\right) \cdot V\left(t_{0}\right)+\sup _{t_{0} \leq \theta \leq t} \chi\left(\|\dot{\boldsymbol{\tau}}(\theta)\|^{2}\right) .
$$

The following lemma is key to proving the convergence of the decision-making algorithm in Theorem 1:

Lemma 1. Let the dynamics (5) of the decision variables $x_{i}$ start from $x_{i}(0)=1$, with gains updated by (6)-(7) and $\alpha_{i}(0)=0, \forall i=1, \cdots, N_{s}$. Then, the dynamic modulations (12) and (13), of $h_{i j}(t)$ and $w_{i j}(t)$, guarantee that:

1. The states $\mathrm{x}$ and $\boldsymbol{\delta}$ stay in the invariant set:

$$
\mathcal{I}=\left\{\left(\|\mathbf{x}\|^{2}-N_{s}\right)^{2}+2 \epsilon\|\boldsymbol{\delta}\|^{2} \leq 2 N_{s}^{2} \epsilon \bar{\tau}^{2}+\frac{N_{s} \epsilon \dot{\bar{\tau}}}{2 \sigma^{2}}\right\},
$$

where $\overline{\dot{\tau}}$ is the upper bound of $\dot{\tau}_{i}$;

2. If $\epsilon$ is selected by:

$$
\epsilon \leq 2 \sigma^{2}\left(N_{s}-\rho\right)^{2} /\left(4 N_{s}^{2} \sigma^{2} \bar{\tau}^{2}+N_{s} \overline{\dot{\tau}}\right)
$$

with $0<\rho<N_{s}$, then $\mathbf{x}$ and $\delta$ exponentially converge to the attractive set:

$$
\mathcal{A}=\left\{\left(\|\mathbf{x}\|^{2}-N_{s}\right)^{2}+2 \epsilon\|\boldsymbol{\delta}\|^{2} \leq \frac{N_{s} \epsilon \overline{\dot{\tau}}}{2 \sigma^{2}}\right\}
$$

and the rate of convergence is $\sigma \rho$.

Proof. 1. Let $t_{0}=0$. The definition of $V$ (11) and (15) yield:

$$
\left(\|\mathbf{x}\|^{2}-N_{s}\right)^{2}+2 \epsilon\|\boldsymbol{\delta}\|^{2} \leq 4 V(0)+\frac{N_{s} \epsilon \bar{\tau}}{2 \sigma^{2}} .
$$

The initializations $x_{i}(0)=1$ and $\alpha_{i}(0)=0$ guarantee that:

$$
V(0)=\frac{\epsilon}{2} \cdot \boldsymbol{\tau}^{\top}(0) \cdot \overline{\mathbf{L}}_{c} \cdot \overline{\mathbf{L}}_{c} \cdot \boldsymbol{\tau}(0) \leq \frac{N_{s}^{2} \epsilon \bar{\tau}^{2}}{2},
$$

because 0 and $N_{s}$ are eigenvalues of $\overline{\mathbf{L}}_{c}$. Together, Equations (17)-(18) imply that $\mathrm{x}$ and $\boldsymbol{\delta}$ are bounded within $\mathcal{I}$.

2. The invariant set $\mathcal{I}$ indicates that:

$$
\|\mathbf{x}\|^{2} \geq N_{s}-\sqrt{2 N_{s}^{2} \epsilon \bar{\tau}^{2}+\frac{N_{s} \epsilon \overline{\dot{\tau}}}{2 \sigma^{2}}} .
$$

The selection of $\epsilon$ (16) then guarantees that $\|\mathbf{x}\|^{2} \geq \rho$ and thus $\kappa(t) \geq \sigma \rho$ for all time. By (17), it follows that:

$$
\begin{aligned}
& \left(\|\mathbf{x}\|^{2}-N_{s}\right)^{2}+2 \epsilon\|\boldsymbol{\delta}\|^{2} \\
\leq & 4 \exp (-\sigma \rho t) \cdot V(0)+4 \sup _{0 \leq \theta \leq t} \chi\left(\|\dot{\boldsymbol{\tau}}(\theta)\|^{2}\right),
\end{aligned}
$$

which proves the exponential convergence to $\mathcal{A}$.
The following theorem summarizes the convergence of the proposed decision-making algorithm:

Theorem 1. Let the distributed decision-making layer (5)-(7) wih time-varying encodings $\tau_{i}$ of user inputs be initialized by $x_{i}=1$ and $\alpha_{i}=0$, and let $\Delta_{\tau}>0$ be a preset resolution of the decision-making algorithm. Further: (i) choose $\sigma$ by:

$$
\sigma \geq \sqrt{N_{s} \bar{\tau}} /\left(p \Delta_{\tau}\right)
$$

where $p<1$; (ii) select $\epsilon$ by (16); and (iii) update the parameters $h_{i j}(t)$ by (12) and $w_{i j}(t)$ by (13) for all $i \sim j$. Then, if there exists $k$ such that $\tau_{k}(t)>\Delta_{\tau}>\tau_{i}(t)>0$ for all $t \geq t_{0}=0$ and for all $i=1, \cdots, N_{l s}$ with $i \neq k$, the decision variable $x_{k}(t)$ asymptotically converges to the positive limit set:

$$
\mathcal{W}_{k}=\left\{x_{k} \geq w=\max \left(N_{s}-p \Delta_{\tau} \sqrt{\epsilon / 2}, \sqrt{\rho}\right)\right\}
$$

while $x_{i}(t) \rightarrow 0$ asymptotically as $t \rightarrow+\infty$ for all $i \neq k$.

Proof. The selection (20) $\sigma$ leads to:

$$
\sup _{0 \leq \theta \leq t} \chi\left(\|\dot{\boldsymbol{\tau}}(\theta)\|^{2}\right) \leq \epsilon p^{2} \Delta_{\tau}^{2} / 8 .
$$

From (19), the selection (16) of $\epsilon$ further ensures that:

$$
2\|\boldsymbol{\delta}\| \leq \sqrt{8 V(0) / \epsilon} \cdot \exp (-\sigma \rho t / 2)+p \Delta_{\tau} .
$$

The variation of all decision variables is studied using the quotient function:

$$
E=\sum_{i \neq k} \frac{x_{i}}{x_{k}},
$$

where $i$ and $k$ are defined in the theorem. By (5), $x_{k}$ and all $x_{i}$-s are positive for all time as they start from 1 . Further, because $\mathrm{x}$ is bounded by $\mathcal{I}, E$ becomes zero if and only if $x_{i}=0$ for all $i \neq k$. Then, the derivative of $E$ along the dynamics of the decision-making layer is:

$$
\begin{aligned}
\dot{E} & =\sum_{i \neq k}\left[\left(\tau_{i}-\tau_{k}\right) \frac{x_{i}}{x_{k}}+\left(\delta_{k}-\delta_{i}\right) \frac{x_{i}}{x_{k}}\right] \\
& \leq-\left(\Delta_{\tau}-2\|\boldsymbol{\delta}\|\right) \cdot \sum_{i \neq k} \frac{x_{i}}{x_{k}} \\
& \leq\left[-(1-p) \Delta_{\tau}+\sqrt{8 V(0) / \epsilon} \cdot \exp (-\sigma \rho t / 2)\right] \cdot E .
\end{aligned}
$$

Then, time integration of $\dot{E}$ gives that, as $t \rightarrow+\infty$,

$$
\begin{array}{r}
E(t) \leq \exp \left[-(1-p) \Delta_{\tau}\left(t-t_{0}\right)+\sqrt{8 V(0) / \epsilon}\right. \\
\left.\quad \times \int_{t_{0}}^{t} \exp (-\sigma \rho \theta / 2) d \theta\right] \cdot E(0) \\
\leq \exp \left[-(1-p) \Delta_{\tau} t+4 \sqrt{2 V(0) / \epsilon} /(\sigma \rho)\right. \\
\left.\times \exp \left(-\sigma \rho t_{0} / 2\right)\right] \cdot E(0) \rightarrow 0,
\end{array}
$$

which, together with $E(t) \geq 0$, ensures that $E(t) \rightarrow 0$. By $\mathcal{I}$ in Lemma $1, \mathbf{x}$ is bounded, and thus $E(t) \rightarrow 0$ indicates 
that $x_{i}(t) \rightarrow 0$ for every $i \neq k$. Because $\mathrm{x}$ asymptotically converges to $\mathcal{A}, x_{i}(t) \rightarrow 0$ for all $i \neq k$ further means that $x_{k}(t)$ converges to:

$$
\mathcal{W}_{k 1}=\left\{\left(x_{k}^{2}-N_{s}\right)^{2} \leq N_{s} \epsilon \bar{\tau} /\left(2 \sigma^{2}\right) \leq \epsilon p^{2} \Delta_{\tau}^{2} / 2\right\} .
$$

Further, the proof of the second item of Lemma 1 shows that (16) makes $\|\mathbf{x}\|^{2} \geq \rho$. Because $x_{k}$ and all $x_{i}$-s remain positive throughout time, it follows that:

$$
x_{k}(t)+\sum_{i \neq k} x_{i}(t) \geq\|\mathbf{x}(t)\| \geq \sqrt{\rho} .
$$

As a result, $x_{i}(t) \rightarrow 0$ for every $i \neq k$ indicates that $x_{k}(t)$ also approaches:

$$
\mathcal{W}_{k 2}=\left\{x_{k} \geq \sqrt{\rho}>0\right\} .
$$

Thus, $x_{k}(t)$ asymptotically converges to $\mathcal{W}_{k} \supseteq \mathcal{W}_{k 1} \cap \mathcal{W}_{k 2}$.

The convergence of the proposed decision-making strategy for the case that the winner operator commands multiple slaves can be proven similarly and is omitted here due to page limit constraints. By Theorem 1, the decision variables of active slaves receiving commands from the winner converge to a positive limit set while the decision variables of all other slave approach zero. By comparing its decision variable $x_{i}$ to a threshold $x<w$, each slave $i$ can decide if it receives commands from the winner: yes if $x_{i} \geq y$ and no otherwise.

\section{EXPERIMENTAL RESULTS}

Figure 2 shows the distributed 3-masters-11-slaves teleoperation testbed that serves to validate the performance of the proposed decision-making algorithm. It includes: 3 black Novint Falcon robots, the master devices, operated by 3 volunteers; 4 Geomagic Touch robots, the active slaves, receiving user commands from the masters; and 7 white Novint Falcon robots, the asleep slaves, that communicate only with other slaves. Each robot is locally controlled via USB 2.0 by a $\mathrm{C}++$ program on a dedicated Linux machine. All Linux machines access the Internet using a 16-port network switch (with bandwidth $32 \mathrm{Gbps}$ ), and run the Robot Operating System (ROS) to support distributed robot communications. In Figure 2, the unidirectional dashed arrows depict the transmissions of user commands from masters to active slaves, and the bidirectional solid arrows indicate the information exchanges among the slaves. Robot sensing, communication and control run at $1 \mathrm{kHz}$. The additional computer at the bottom of Figure 2 records the experimental data at $100 \mathrm{~Hz}$ and displays the movements of the end-effectors of all slave robots.

The experiment has two phases: (i) during the first 5 minutes, users apply forces to the slave group sequentially, starting with user 3 and ending with user 1; (ii) during the next 3.5 minutes, they apply forces to the slave network simultaneously. Figure 3-Figure 5 plot the time histories of all operator forces. The shaded areas in these figures indicate time intervals

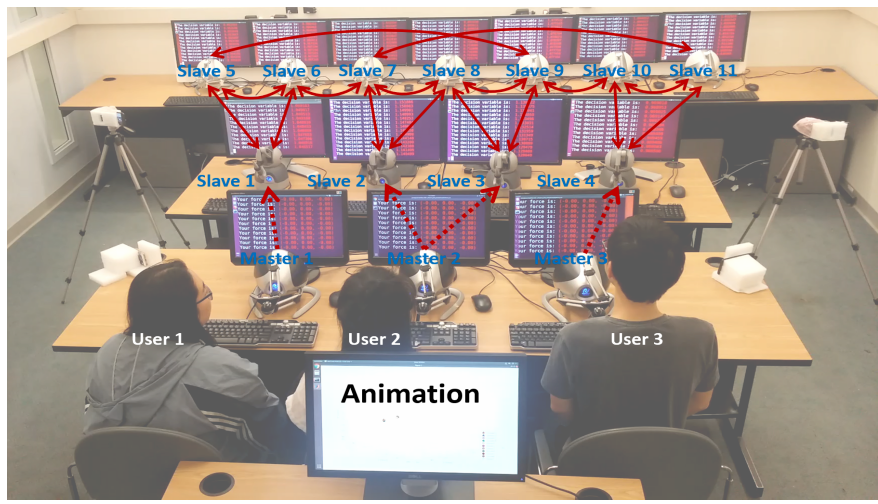

Figure 2. The distributed 3-masters-11-slaves teleoperation testbed.
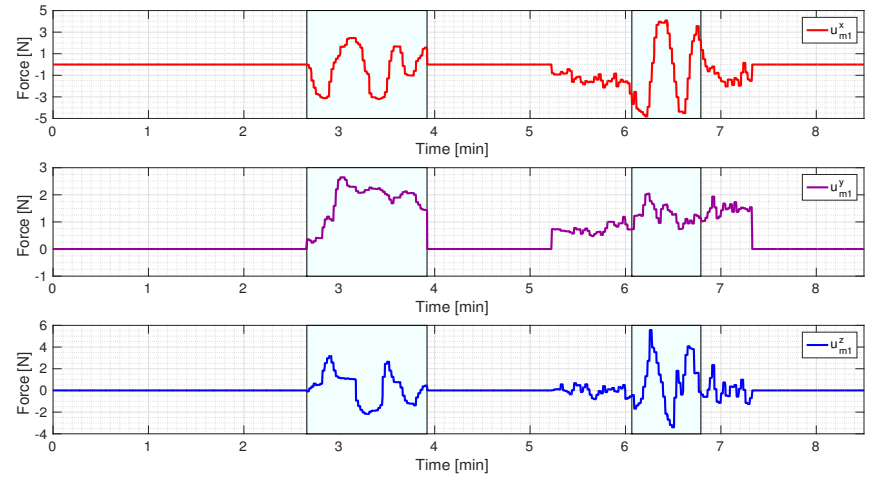

Figure 3. The force command $\mathbf{u}_{m 1}$ of user 1 along $x$-, $y$-, and $z$-axes.
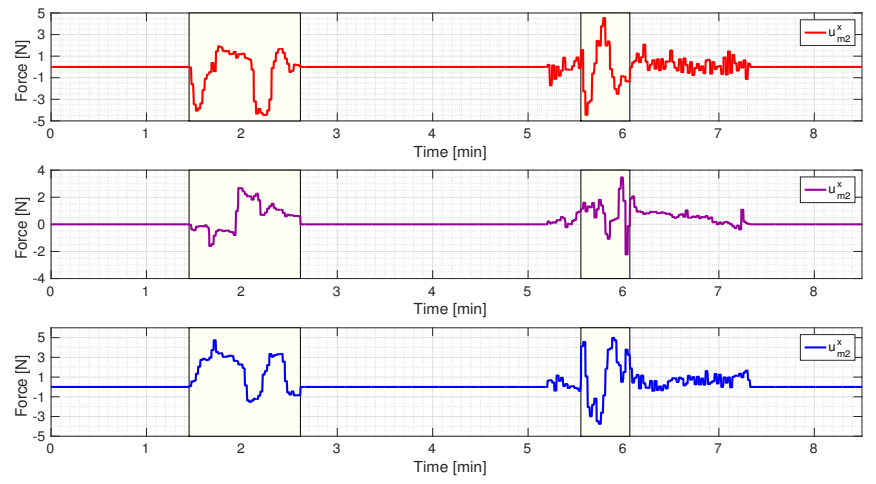

Figure 4. The force command $\mathbf{u}_{m 2}$ of user 2 along $x$-, $y$-, and $z$-axes.

when the corresponding users apply the largest force among all users.

The encodings of user commands to the decision-making layer are compared in Figure 6. In this figure, $\tau_{2}$ and $\tau_{3}$ overlap because user 2 applies their force to two active slaves, 2 and 3 . In the first phase, users 3, 2 and 1 apply forces sequentially in this order, and the encodings $\tau_{4}, \tau_{3}\left(\tau_{2}\right)$, and $\tau_{1}$ become larger than 1 during the time intervals $T_{1}, T_{2}$ and $T_{3}$, respectively. In the second phase, all users apply forces simultaneously, so all encodings become larger than 1 during all time intervals $T_{4}, T_{5}, T_{6}$ and $T_{7}$. However, only one user applies the largest 

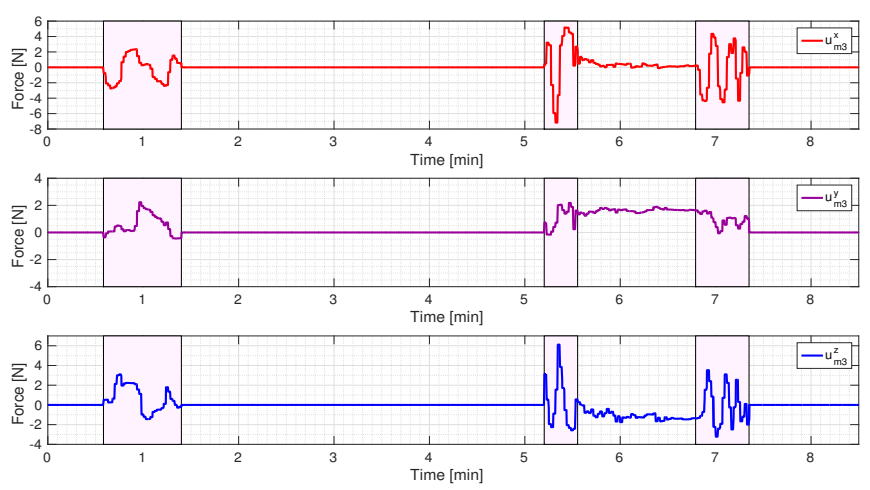

Figure 5. The force command $\mathbf{u}_{m 3}$ of user 3 along $x$-, $y$-, and $z$-axes.

force during each of these time intervals, see the command encodings in Figure 6.

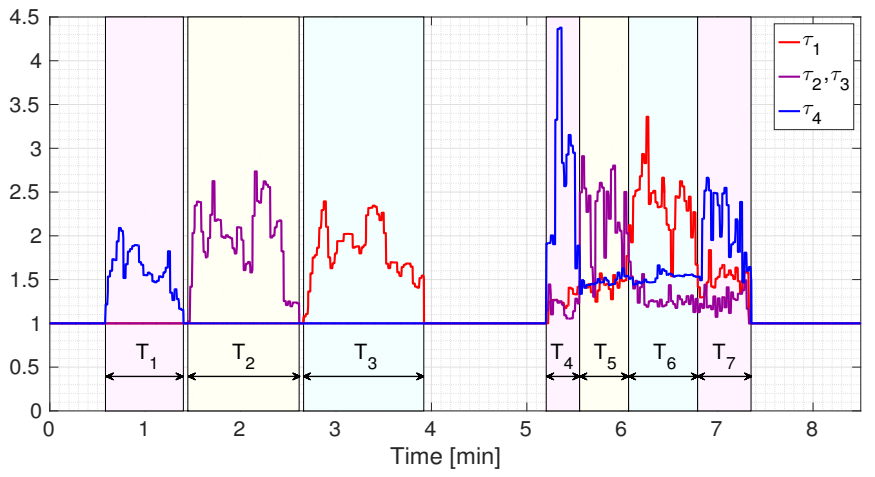

Figure 6. The encodings of user commands to the decision-making layer.

Due to delays of up to $36 \mathrm{~ms}$ in the experimental data transmissions, the damping $-10 \alpha_{i}$ is injected in (7) to practically stabilize the decision-making algorithm, and stabilization by damping injection is currently under theoretical study. The time histories of the practically stabilized decision variables are depicted in Figure 7 for the active slaves, and in Figure 8 for the asleep slaves. Figure 7 shows that the decision variables of active slaves grow larger than 2 when the active slaves receive force commands from the winner. Further, Figure 7Figure 8 illustrate that: (i) the decision variables of all slaves approach 0 unless they receive force commands from the winner; and (ii) if the slave network receives no user command, i.e., $\tau_{i}=1$ for all $i$, then all decision variables return to 1 . Therefore, the threshold for winner selection is set to 2. Lastly, all decision variables $x_{i}$ stop decreasing when they reach 0.001 to avoid zero crossings in the discrete time implementation of the decision-making algorithm.

\section{CONCLusions}

This paper has presented a distributed decision-making strategy for winner-take-all teleoperation of multi-robot systems. The strategy equips all slave robots with a decision variable which it adjusts based on the variations (i) of the
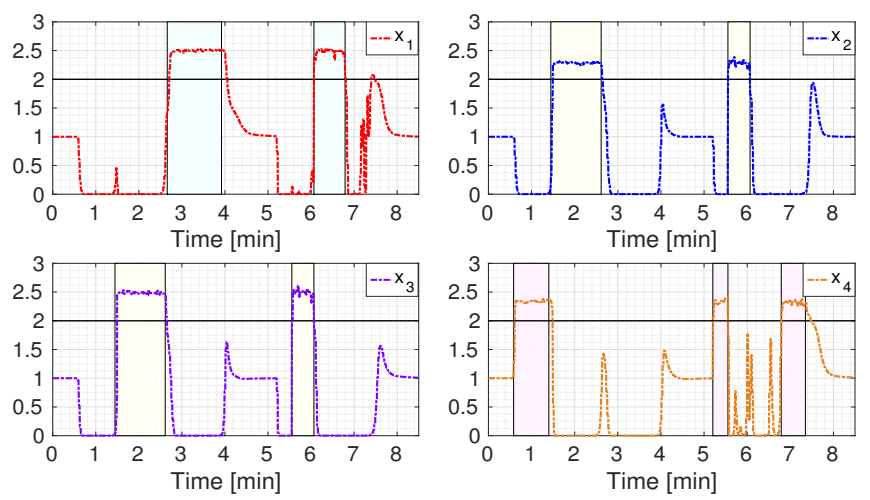

Figure 7. The decision variables of the active slave robots.

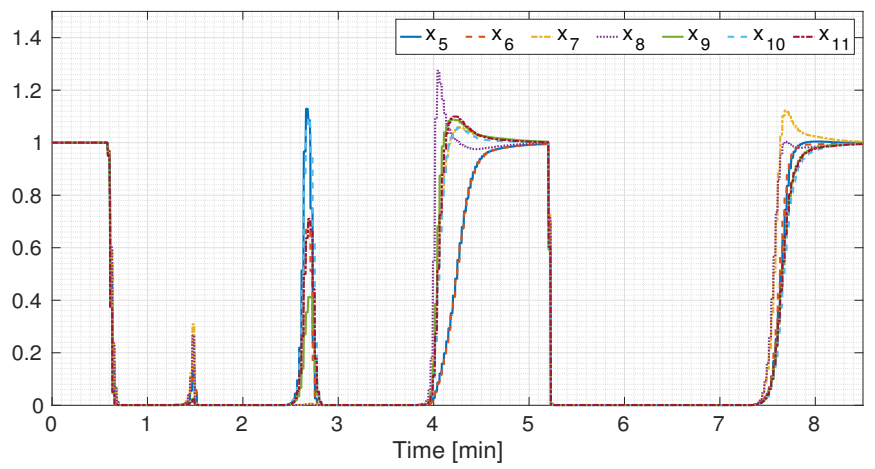

Figure 8. The decision variables of the asleep slave robots.

force command received from the local operator and (ii) of the decision variables of the neighbouring slaves. Lyapunov stability analysis shows that the proposed strategy can drive the decision variables of the active slaves who receive force commands from the winner to a positive limit set while reducing other decision variables to zero. It thus enables the slave group to identify the winner in a decentralized manner. An experimental winner-take-all teleoperation in a distributed 3-masters-11-slaves teleoperation testbed has illustrated the efficacy of the proposed decision-making strategy. Upcoming research seeks to make the algorithm robust to time-varying communication delays.

\section{REFERENCES}

[1] Q. M. Ta, S. Lyu, and C. C. Cheah, "Human-guided optical manipulation of multiple microscopic objects," in 2018 IEEE International Conference on Robotics and Automation. IEEE, 2018, pp. 1-5.

[2] C. Secchi, S. Stramigioli, and C. Fantuzzi, Control of interactive robotic interfaces: A port-Hamiltonian approach. Springer Science \& Business Media, 2007, vol. 29.

[3] M. Selvaggio, P. Robuffo Giordano, F. Ficuciellol, and B. Siciliano, "Passive task-prioritized shared-control teleoperation with haptic guidance," in 2019 IEEE International Conference on Robotics and Automation. IEEE, 2019, pp. 430-436.

[4] E. Sartori, C. Tadiello, C. Secchi, and R. Muradore, "Tele-echography using a two-layer teleoperation algorithm with energy scaling," in 2019 IEEE International Conference on Robotics and Automation. IEEE, 2019 , pp. $1569-1575$. 
[5] D. Lee, A. Franchi, P. R. Giordano, H. I. Son, and H. H. Bülthoff, "Haptic teleoperation of multiple unmanned aerial vehicles over the internet," in 2011 IEEE International Conference on Robotics and Automation. IEEE, 2011, pp. 1341-1347.

[6] A. Franchi, C. Secchi, H. I. Son, H. H. Bülthoff, and P. R. Giordano, "Bilateral teleoperation of groups of mobile robots with time-varying topology," IEEE Transactions on Robotics, vol. 28, no. 5, pp. 10191033, 2012.

[7] P. R. Giordano, A. Franchi, C. Secchi, and H. H. Bülthoff, "A passivitybased decentralized strategy for generalized connectivity maintenance," The International Journal of Robotics Research, vol. 32, no. 3, pp. 299323, 2013.

[8] A. Y. Mersha, S. Stramigioli, and R. Carloni, "On bilateral teleoperation of aerial robots," IEEE Transactions on Robotics, vol. 30, no. 1, pp. 258-274, 2013.

[9] L. Sabattini, C. Secchi, and C. Fantuzzi, "Controlling the interaction of a multi-robot system with external entities," in 2018 IEEE International Conference on Robotics and Automation. IEEE, 2018, pp. 7654-7659.

[10] C. Secchi and F. Ferraguti, "Energy optimization for a robust and flexible interaction control," in 2019 IEEE International Conference on Robotics and Automation. IEEE, 2019, pp. 1919-1925.

[11] M. Minelli, F. Ferraguti, N. Piccinelli, R. Muradore, and C. Secchi, "An energy-shared two-layer approach for multi-master-multi-slave bilateral teleoperation systems," in 2019 IEEE International Conference on Robotics and Automation. IEEE, 2019, pp. 423-429.

[12] S. S. Groothuis and S. Stramigioli, "Energy budget transaction protocol for distributed robotic systems," in 2019 IEEE International Conference on Robotics and Automation. IEEE, 2019.

[13] M. Kwon, M. Li, A. Bucquet, and D. Sadigh, "Influencing leading and following in human-robot teams," in Proceedings of Robotics: Science and Systems, FreiburgimBreisgau, Germany, 2019.

[14] Y. Sung, A. K. Budhiraja, R. K. Williams, and P. Tokekar, "Distributed simultaneous action and target assignment for multi-robot multi-target tracking," in 2018 IEEE International conference on robotics and automation. IEEE, 2018, pp. 1-9.

[15] S. Ghapani, S. Rahili, and W. Ren, "Distributed average tracking of physical second-order agents with heterogeneous unknown nonlinear dynamics without constraint on input signals," IEEE Transactions on Automatic Control, vol. 64, no. 3, pp. 1178-1184, 2019.

[16] J. George and R. Freeman, "Robust dynamic average consensus algorithms," IEEE Transactions on Automatic Control, 2019.

[17] J. D. Peterson, T. Yucelen, J. Sarangapani, and E. L. Pasiliao, "Activepassive dynamic consensus filters with reduced information exchange and time-varying agent roles," IEEE Transactions on Control Systems Technology, 2019.

[18] K. I. Diamantaras and S. Y. Kung, Principal Component Neural Networks: Theory and Applications. New York, NY, USA: John Wiley \& Sons, Inc., 1996.

[19] S. Li, M. Zhou, X. Luo, and Z. You, "Distributed winner-take-all in dynamic networks," IEEE Transactions on Automatic Control, vol. 62, no. 2, pp. 577-589, 2017.

[20] D. Zhou, Z. Wang, and M. Schwager, "Agile coordination and assistive collision avoidance for quadrotor swarms using virtual structures," IEEE Transactions on Robotics, vol. 34, no. 4, pp. 916-923, 2018.

[21] M. Mesbahi and M. Egerstedt, Graph theoretic methods in multiagent networks. Princeton University Press, 2010. 